\title{
Curie temperature of Kondo lattice films with finite itinerant charge carrier density
}

\author{
J. Kienert and W. Nolting \\ Festkörpertheorie, Institut für Physik, Humboldt-Universität zu Berlin, Newtonstr. 15, 12489 Berlin, Germany
}

\begin{abstract}
We present a model study of ferromagnetic films consisting of free Bloch electrons coupled to localized moments (Kondo lattice films). By mapping the local interaction onto an effective Heisenberg Hamiltonian we obtain temperature and carrier density dependent exchange integrals mediating the interaction between local moments via the conduction electrons. The non-perturbative approach recovers analytically the weak-coupling RKKY interaction and yields convincing numerical results in the strong coupling (double exchange) regime. The Curie temperature is calculated for various coupling strengths, band fillings, and numbers of layers. The results are compared with total energy calculations. We discuss the influence of charge transfer between film layers and of anisotropy on the Curie temperature. The model we investigate is considered relevant for the understanding of the basic magnetic properties of manganites, diluted magnetic semiconductors, and rare earth substances as, e.g., Gadolinium.
\end{abstract}

\section{INTRODUCTION}

The Kondo lattice model (KLM) is one of the prototype models in solid state physics whenever the coupling of itinerant charge carrier spins with immobile localized magnetic moments has to be considered. There are various quite different classes of materials for which the KLM is used to describe the electronic and magnetic properties at least in principle.

In its simplest single-band version the KLM Hamiltonian reads

$$
H=\sum_{i j \sigma}\left(T_{i j}-\mu \delta_{i j}\right) c_{i \sigma}^{\dagger} c_{j \sigma}-J \sum_{i} \mathbf{S}_{i} \cdot \boldsymbol{\sigma}_{i}
$$

The first term describes non-interacting electrons $\left(c_{i \sigma}^{(\dagger)}\right.$ annihilates (creates) an electron of spin $\sigma=\uparrow, \downarrow$ at lattice site $i$ ) with hopping integrals $T_{i j} . \boldsymbol{\sigma}_{i}$ is the electron spin and $\mathbf{S}_{i}$ the localized spin operator, and both are exchange coupled by $J$.

Depending on the sign of $J$ one is confronted with quite different physics. Numerous studies have been carried to understand the physics of heavy fermion systems. Here the coupling between electrons and localized spins favors an antiparallel configuration $(J<0)$ leading to a competition between RKKY interaction and spin screening effects.

In this work we want to focus on a favored parallel spin alignment $(J>0$, ferromagnetic Kondo lattice model, FKLM). The FKLM was introduced to model the magnetism in some manganese compounds ("manganites"). In the framework of a simple two-site model this kind of interaction was found to result from the so-called double exchange lending its name to the lattice version in the strong coupling regime $\frac{1,2}{1}$

In the manganites the $5 \mathrm{Mn}$ d-shells are split by the crystal field into three degenerate $t_{2 g}$-orbitals forming localized spins of $S=\frac{3}{2}$ which interact via Hund's rule with itinerant electrons stemming from the remaining two degenerate $e_{g}$-orbitals. Due to the Jahn-Teller effect and the strong Coulomb interaction among the conduction electrons the manganites exhibit a complex phase diagram, the most prominent phenomenon of which is colossal magnetoresistance ${ }^{3.4}$ Although for a more quantitative description of manganite materials additional ingredients like orbital physics, electron-polaron interaction, and a strong Hubbard-like Coulomb interaction are needed, numerous research work on the subject has been carried out using the simple one-orbital model (1) ${ }^{\underline{3}}$

More recently attention was directed to the diluted magnetic semiconductors (DMS) that are thought to have the potential for promising technical applications

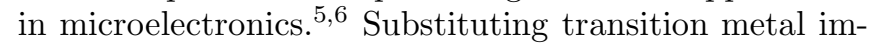
purities like $\mathrm{Mn}$ in a semiconducting host (often III/V like GaAs) localized magnetic moments are introduced with the consequence of ferromagnetic ordering of the randomly distributed maganese cation spins that interact via their (ferromagnetic, intermediate) coupling to valence and impurity band holes.

Another important field of application of the FKLM is the theoretical description of rare earth substances like Eu and Gd and their compounds. Here it initially served to explain the famous red-shift of the absorption edge of the optical $4 f-5 d$ transition in the ferromagnetic

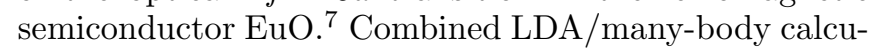
lations based on the FKLM yielded realistic values of the Curie temperature for $\mathrm{Gd}^{8}$ and predicted surface states in $\mathrm{EuO}-$ films ${ }^{9}$. Doping Gd into $\mathrm{EuO}$ makes for an attractive means to tune the charge carrier density and thus the carrier-induced coupling among the magnetic ions $\underline{10}$ Having the same benefit, Gd-doped GaN was reported to yield high $T_{C}$ ferromagnetism above $300 \mathrm{~K}, 11$

In all of the above-mentioned substances, especially regarding possible technical applications in the future, it is important to understand and make use of the special physical properties due to reduced dimensionality. Several recent works focussed on the role of magnetic anisotropy effects in thin films of manganites 12 or of DMS films like $(\mathrm{Ga}, \mathrm{Mn}) \mathrm{As}_{\mathbf{s}} \underline{13}$. Gd films are investigated due to their potential employment in spintronics as well as motivated by the "evergreen" issue of a possibly enhanced surface Curie temperature.14,15,16 It is furthermore a wellknown fact that the Curie temperature strongly depends on the film thickness. Due to the broad relevance of the 
FKLM in current research on magnetism and electronic correlations and due to the importance of film structures it is worthwhile investigating the dependence of the ferromagnetic transition temperature on the model parameters.

From the theoretical point of view our treatment of FKLM films that is divided up into two parts. First we solve the problem for the electronic self-energy within an equation of motion approach that is based on a decoupling scheme fulfilling non-trivial limiting cases. In the second step we map the interaction onto an effective localized spin Hamiltonian by integrating out the electronic degrees of freedom. This results in effective exchange integrals which incorporate the interaction of the localized moments via the conduction electrons beyond conventional RKKY theory. They depend on temperature and carrier density. In sum we end up with a self-consistent theory which does not require the assumption of classical core spins and which is employable at all temperatures.

Results on the magnetization and critical temperature of ferromagnetic KLM model films with finite band occupation have already been reported in Ref. 17. However, in the present work we make use of a more sophisticated approach for the fermionic subsystem and carry out a wider and considerably more detailed investigation of the Curie temperature. A similar system is also treated in Ref. 18 based on a method guided by the same spirit. However, the theory of the electronic subsystem there is on a mean-field level only and just nearest neighbor Heisenberg interaction is considered, in spite of the underlying RKKY-like exchange being long ranged. What's more the effective exchange integrals derived in Ref. 18 in terms of the susceptibility of free electrons are proportional to $J^{2}$ and thus fail to give the appropriate strong-coupling behavior where the Curie temperature is independent of $J$. As far as the numerical evaluation is concerned the thickness dependence of the Curie temperature is analysed.

This paper is organized as follows. In the next section we briefly summarize our method to solve the KLM for the electronic self-energy. Then we derive an effective Heisenberg Hamiltonian which results from integrating out the fermionic degrees of freedom. In the numerical results section we discuss the temperature dependent quasiparticle excitation spectrum and charge transfer before we present an extensive analysis of the Curie temperature and its dependence on the various model paramters like, e.g., the magnetic anisotropy strength.

\section{ELECTRONIC SELF-ENERGY}

In second-quantized notation and introducing Greek indices to number the layers the Hamiltonian (1) becomes

$$
\begin{aligned}
H & =H_{0}+H_{J} \\
& =\sum_{\substack{i j \sigma \\
\alpha \beta}}\left(T_{i j}^{\alpha \beta}-\mu \delta_{i j}^{\alpha \beta}\right) c_{i \alpha \sigma}^{\dagger} c_{j \beta \sigma} \\
& -\frac{1}{2} J \sum_{i \alpha \sigma}\left(z_{\sigma} S_{i \alpha}^{z} n_{i \alpha \sigma}+S_{i \alpha}^{-\sigma} c_{i \alpha \sigma}^{\dagger} c_{i \alpha-\sigma}\right) .
\end{aligned}
$$

$S_{i \alpha}^{+,-}=S_{i \alpha}^{x} \pm i S_{i \alpha}^{y}, \uparrow(\downarrow)=+(-), z_{\uparrow}=1, z_{\downarrow}=-1$, and $S_{i \alpha}^{x, y, z}$ are the cartesian components of the localized spin operator $\mathbf{S}_{i \alpha} . n_{i \alpha \sigma}$ is the particle density operator.

In the following we apply an equation of motion (EOM) approach based on a decoupling scheme already applied to semiconducting Kondo lattice films $\left(\mathrm{EuO}^{9}\right.$ and $\mathrm{EuS}^{19}$ ) and to bulk $\mathrm{Gd}^{8}$ in combined LDA and many-body calculations (moment conserving decoupling approximation, MCDA). For details of the method we refer the reader to corresponding model studies ${ }^{20,21,22}$ After writing down the EOM of the one-particle Green function $G_{i j \sigma}^{\alpha \beta}(E)=\left\langle\left\langle c_{i \alpha \sigma} ; c_{j \beta \sigma}^{\dagger}\right\rangle\right\rangle$ and the generated higher Green functions a decoupling is performed that is guided by some non-trivial cases, as, e.g., the ferromagnetic semiconductor at $\mathrm{T}=0$. In the $\mathbf{k}$-space defined within the film plane and using matrix notation one can write:

$$
\mathbf{G}_{\mathbf{k} \sigma}(E)=\left[E \mathbf{I}-\epsilon(\mathbf{k})-\boldsymbol{\Sigma}_{\mathbf{k} \sigma}(E)\right]^{-1} .
$$

$\epsilon(\mathbf{k})$ is the hopping matrix. A local approximation of the self-energy $\Sigma_{i j \sigma}^{\alpha \beta}(E) \rightarrow \delta_{i j} \delta_{\alpha \beta} \Sigma_{\alpha \sigma}(E)$ is performed. This corresponds to neglecting magnon energies in the threedimensional $\operatorname{case}^{23}$ and is justified by the fact that these energies are usually orders of magnitude smaller than the exchange coupling $J$ and the electron bandwidth $W$.

From (5) one immediately obtains the one-particle local density of states (LDOS):

$$
\rho_{\alpha \sigma}(E)=-\frac{1}{\pi \hbar} \operatorname{Im} G_{i i \sigma}^{\alpha \alpha}\left(E+i 0^{+}-\mu\right) .
$$

It should be mentioned that in general the MCDA approach does not capture Kondo scaling and especially violates the Luttinger theorem $\operatorname{Im} \Sigma_{\sigma}\left(E=\epsilon_{F}\right) \rightarrow 0{ }^{24}$ Although this might well be due to our approximative scheme questions if and in which parameter regimes the ferromagnetic Kondo lattice model is a Fermi liquid still remain open. Nevertheless we would consider it worthwhile to investigate the low temperature Kondo physics within the framework of our theory. In the present work, however, we rather want to focus on the magnetic transition temperature.

The self-energy matrix $\boldsymbol{\Sigma}_{\sigma}(E)$ depends on various expectation values of pure fermionic, mixed fermionic-spin, and pure localized spin character:

$$
\begin{aligned}
\Sigma_{\sigma}^{\alpha}= & F\left(\left\langle n_{\alpha \sigma}\right\rangle,\left\langle S_{\alpha}^{-\sigma} c_{\alpha \sigma}^{\dagger} c_{\alpha-\sigma}\right\rangle,\left\langle S_{\alpha}^{z} n_{\alpha \sigma}\right\rangle,\right. \\
& \left.\left\langle S_{\alpha}^{z}\right\rangle,\left\langle\left(S_{\alpha}^{z}\right)^{2}\right\rangle,\left\langle\left(S_{\alpha}^{z}\right)^{3}\right\rangle,\left\langle S_{\alpha}^{+} S_{\alpha}^{-}\right\rangle\right) .
\end{aligned}
$$


The first two types can be calculated self-consistently within the MCDA using the corresponding Green functions and the spectral theorem 25 . The localized spin correlation functions like, to lowest order, the layerdependent magnetization $\left\langle S_{\alpha}^{z}\right\rangle$ need further consideration. In order to evaluate these we map the interaction term (44) onto an effective coupling between the localized spins, which is carried out in the next section.

\section{EFFECTIVE LOCALIZED SPIN HAMILTONIAN}

It is well known that using perturbation theory (4) leads to the so-called RKKY interaction. ${ }^{26,27}$ This second order interaction between localized spins is long ranged and yields an oscillatory behavior of the exchange coupling which is mediated by uncorrelated electrons. It appears highly desirable to have such a mapping without being restricted to the weak coupling regime. This requires finding an effective Hamiltonian

$$
\begin{aligned}
H_{J}^{e f f} & =\left\langle H_{J}\right\rangle^{(c)} \\
& =-\frac{J}{2 N} \sum_{\substack{i \alpha \sigma \sigma^{\prime} \\
\mathbf{k q}}} e^{-i \mathbf{q} \mathbf{R}_{i}^{\alpha}}\left(\mathbf{S}_{i \alpha} \cdot \boldsymbol{\sigma}\right)_{\sigma \sigma^{\prime}}\left\langle c_{\mathbf{k}+\mathbf{q} \alpha \sigma}^{\dagger} c_{\mathbf{k} \alpha \sigma^{\prime}}\right\rangle^{(c)},
\end{aligned}
$$

where $N$ is the number of lattice sites in one layer and the superscript $c$ formally indicates that the averaging is performed in the fermionic subspace only. The method we present in this section has been applied before to bulk Kondo lattice systems $\frac{8,22}{2}$ We generalize it into a matrix formulation which allows us to consider film structures, but is also relevant whenever sublattice decompositions are necessary, for instance when dealing with antiferromagnetic configurations.

In order to obtain the expectation value in (8) we introduce the modified Green function

$$
\hat{G}_{\mathbf{k}, \mathbf{k}+\mathbf{q} \alpha \beta}^{\sigma^{\prime} \sigma}(E)=\left\langle\left\langle c_{\mathbf{k} \alpha \sigma^{\prime}} ; c_{\mathbf{k}+\mathbf{q} \beta \sigma}^{\dagger}\right\rangle\right\rangle^{(c)} .
$$

The EOM for $\hat{G}$ reads

$$
\begin{array}{r}
\sum_{\gamma}\left(E \delta_{\gamma \alpha}-\epsilon_{\mathbf{k}}^{\alpha \gamma}\right) \hat{G}_{\mathbf{k}, \mathbf{k}+\mathbf{q} \gamma \beta}^{\sigma^{\prime} \sigma}(E)=\delta_{\mathbf{q} 0} \delta_{\sigma \sigma^{\prime}} \delta_{\alpha \beta} \\
-\frac{J}{2 N} \sum_{i \mathbf{p} \sigma^{\prime \prime}} e^{i(\mathbf{k}-\mathbf{p}) \mathbf{R}_{i}^{\alpha}}\left(\mathbf{S}_{i \alpha} \cdot \sigma\right)_{\sigma^{\prime} \sigma^{\prime \prime}} \hat{G}_{\mathbf{p}, \mathbf{k}+\mathbf{q} \alpha \beta}^{\sigma^{\prime \prime} \sigma}(E) .
\end{array}
$$

In principle, this equation can be solved iteratively. Applying the spectral theorem then yields the expectation value in (8). This correlation function is an operator since we are working in the fermionic subspace, resulting in an effective interaction among the localized spins $\mathbf{S}_{i}$ only.

We have to find a manageable approximation for $\hat{G}$ on the right hand side of (10). It can easily be shown that in the non-interacting limit,

$$
\hat{G}_{\mathbf{p}, \mathbf{k}+\mathbf{q} \alpha \beta}^{\sigma^{\prime \prime} \sigma}(E) \rightarrow \delta_{\sigma^{\prime \prime} \sigma} \delta_{\mathbf{p}, \mathbf{k}+\mathbf{q}} G_{\mathbf{k}+\mathbf{q} \alpha \beta}^{(0)}(E),
$$

conventional RKKY interaction is reproduced. In order to include spin scattering effectively we dress the free propagator in (12) and replace it by the full propagator $G_{\mathbf{k}+\mathbf{q} \alpha \beta \sigma}(E)$. Within this modified RKKY approximation (MRKKY) the exchange integrals in the effective Hamiltonian

$$
H_{J}^{e f f}=-\sum_{i j \alpha \beta} \hat{J}_{i j}^{\alpha \beta} \mathbf{S}_{i \alpha} \mathbf{S}_{j \beta}
$$

are given by

$$
\hat{J}^{\alpha \beta}(\mathbf{q})=\frac{J^{2}}{4 \pi N} \sum_{\mathbf{k} \sigma} \operatorname{Im} \int_{-\infty}^{+\infty} d E f(E) G_{\mathbf{k} \sigma}^{(0) \alpha \beta}(E) G_{\mathbf{k}+\mathbf{q} \sigma}^{\alpha \beta}(E)
$$

where $f$ is the Fermi function and the sum is over the first Brillouin zone.

Within the Tyablikov approximation 27,28 the imaginary part of the transversal layer-diagonal spin Green function (spectral density) can be written as

$$
-\frac{1}{\pi} \operatorname{Im}\left\langle\left\langle S_{\mathbf{k} \alpha}^{+} ; S_{(-\mathbf{k}) \alpha}^{-}\right\rangle\right\rangle=2\left\langle S_{\alpha}^{z}\right\rangle \sum_{\gamma} \eta_{\mathbf{k}}^{\alpha \gamma} \delta\left(E-E_{\gamma}(\mathbf{k}) \gamma^{\prime} 15\right)
$$

where the spectral weights $\eta_{\mathbf{k}}^{\alpha \gamma}$ and energy poles $E_{\gamma}(\mathbf{k})$ have to be evaluated numerically. For a monolayer we can drop all Greek indices, $\eta_{\mathbf{k}}=1$, and we get the wellknown spin wave energies

$$
E(\mathbf{k})=2\left\langle S_{z}\right\rangle(\hat{J}(\mathbf{0})-\hat{J}(\mathbf{k})) .
$$

Applying the Callen method 27,29 to superlattices the layer-dependent magnetization,

$$
\left\langle S_{\alpha}^{z}\right\rangle=\frac{\left(1+\varphi_{\alpha}+S\right) \varphi_{\alpha}^{2 S+1}+\left(S-\varphi_{\alpha}\right)\left(1+\varphi_{\alpha}\right)^{2 S+1}}{\left(1+\varphi_{\alpha}\right)^{2 S+1}-\varphi_{\alpha}^{2 S+1}}(17)
$$

and other higher order spin correlation functions can be obtained using the Bose-like distribution function

$$
\varphi_{\alpha}=\frac{1}{N} \sum_{\mathbf{k}, \gamma} \frac{\eta_{\mathbf{k}}^{\alpha \gamma}}{e^{\beta E_{\gamma}(\mathbf{k})}-1}
$$

(5), (14), and (17) represent a self-consistent system of equations that can be solved for the one-particle Green function matrix $\mathbf{G}_{\mathbf{k} \sigma}(E)$ and the magnetization $\left\langle S_{\alpha}^{z}\right\rangle$.

Before proceeding to the numerical evaluation we have to reconsider our effective Hamiltonian (13). It is known that for low-dimensional systems anisotropies can become very important and even a necessary condition for magnetic ordering at finite temperature 30,31 We therefore include a single-ion anisotropy term

$$
H_{A}=-K_{2}^{\alpha} \sum_{i \alpha}\left(S_{i \alpha}^{z}\right)^{2}
$$


The physical background of this magnetic anisotropy is spin-orbit coupling, which usually is some order of magnitudes smaller than the exchange coupling between localized spins. A positive $K_{2}^{\alpha}$ favors an out-of-plane easy axis, i.e. perpendicular to the film plane.

We treat this term in the Anderson-Callen approximation $^{32}$ and decouple the higher Green function generated by (19) in the following manner:

$$
\begin{aligned}
&\left\langle\left\langle\left[S_{i \alpha}^{+}, H_{A}\right]_{-} ; S_{j \beta}^{-}\right\rangle\right\rangle=\left\langle\left\langle S_{i \alpha}^{+} S_{i \alpha}^{z}+S_{i \alpha}^{z} S_{i \alpha}^{+} ; S_{j \beta}^{-}\right\rangle\right\rangle \\
& \approx \Phi_{\alpha}\left\langle\left\langle S_{i \alpha}^{+} ; S_{j \beta}^{-}\right\rangle\right\rangle, \\
& \Phi_{\alpha}=2\left\langle S_{\alpha}^{z}\right\rangle\left(1-\frac{1}{2 S^{2}}\left(S(S+1)-\left\langle\left(S_{\alpha}^{z}\right)^{2}\right\rangle\right)\right) .
\end{aligned}
$$

We can drop the site index $i$ due to lateral translational invariance. The single-ion anisotropy acts as an effective field $\Phi_{\alpha}$ coupled to $S_{\alpha}^{z}$. Note that this decoupling is valid only if the magnetization is parallel to the $z$-direction. In order to ensure this a rotation of the coordinate system might be required before the decoupling, for instance when an arbitrary oriented magnetic field is present. 33

For bulk or a monolayer $(\alpha=1)$ it is possible to derive a simple formula for the Curie temperature $T_{C}$. Expanding (17) and (18) in the vicinity of $T_{C}$ one easily finds

$$
\frac{3 k_{B} T_{C}}{S(S+1) N}=\left(\sum_{\mathbf{q}} \frac{1}{\left(K_{2} \gamma+2(\hat{J}(\mathbf{0})-\hat{J}(\mathbf{q}))\right)}\right)_{T=T_{C}}^{-1}
$$

where

$$
\gamma=\lim _{T \rightarrow T_{C}} \frac{\Phi(T)}{\left\langle S^{z}\right\rangle}=\frac{2(2 S-1)}{3 S}
$$

is a constant and depends on the specific decoupling of the anisotropy Green function (20) 34

For $\alpha>1$ the spin wave energies are no longer known in analytical form as in (16). One can then, however, use (17) to determine the critical temperature.

\section{NUMERICAL RESULTS}

All numerical results have been obtained for simple cubic (sc) (100) films. The nearest-neighbor hopping integral $t$ in the tight binding approximation was chosen according to a bulk bandwidth $W=1 \mathrm{eV}$, i.e. $t=-0.083 \mathrm{eV}$. The value of the exchange coupling is assumed to be homogeneous and isotropic for all sites and layers of the films, as already implied by (4). Furthermore for the anisotropy constant we set $K_{2}^{\alpha}=K_{2}$ for all layers. The magnitude of the localized spin is $S=7 / 2$. To keep notation simple we write $n_{(\alpha, \sigma)}$ for the respective expectation values $\left\langle n_{(\alpha, \sigma)}\right\rangle$. $n$ denotes the average electron density.

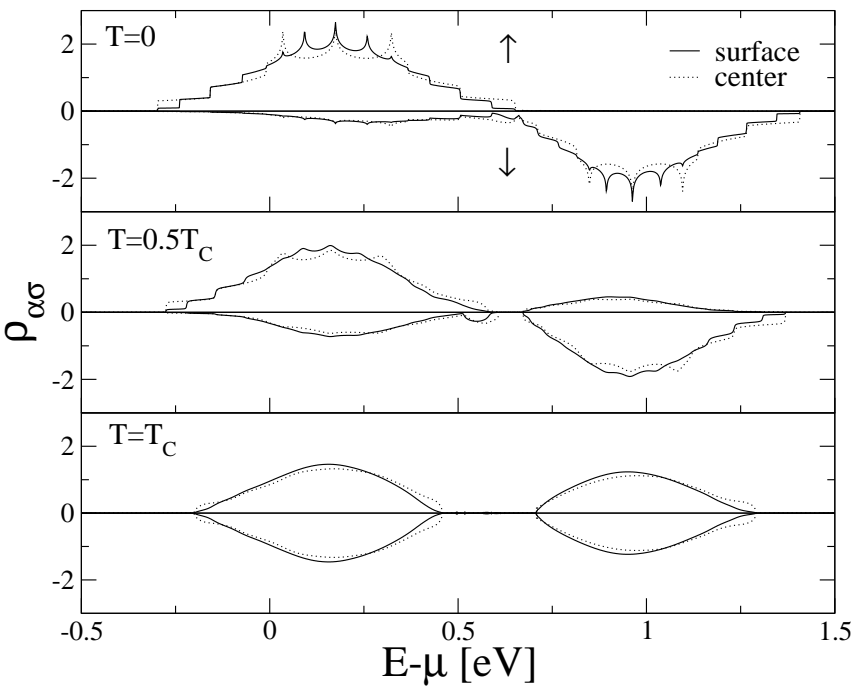

FIG. 1: One-particle density of states of a 5-layer film at different temperatures. Parameters: $n=0.2, J=0.2 \mathrm{eV}, S=$ $7 / 2$. Critical temperature $T_{C}=174 \mathrm{~K}$. Charge neutral calculation.

\section{A. Electronic properties}

\section{One-particle excitation spectrum}

We start with the discussion of the local one-particle density of states. Fig. 1 shows the LDOS at different temperatures between $T=0$ and $T_{C}$. The Curie temperature has been self-consistently computed.

The general picture for a layered system is the same as for the bulk Kondo lattice model. ${ }^{22}$ At low temperature the spin-up spectrum basically consists of the rigidly shifted non-interacting electron energy band. At $T=0$ and in the insulating limit (band occupation $n=0$ ) this is a rigorous result. 25 For the spin-down LDOS the situation is different. Besides a band at higher energies corresponding to an antiparallel coupling of conduction electrons and localized spins there is a scattering part in the energy range of the $\uparrow$-band reflecting the fact that a $\downarrow$-electron can flip its spin by creating a magnon in the localized spin subsystem. With increasing temperature spin symmetry in the spectrum is gradually established. This is accompanied by a reduction of the bandwidth due to reduced effective hopping by spin scattering. For intermediate coupling strengths $J \approx W$ as in Fig. 11 this leads to a temperature-induced opening of a gap in the excitation spectrum. From the zero bandwidth limit one can learn that the distance between the two correlated bands roughly scales as $\sim J\left(S+\frac{1}{2}\right)$.

Apart from these facts already known from bulk results one also observes typical features of reduced dimensionality. The number of van-Hove singularities which are most pronounced at low temperatures is indicative of the number of layers and therefore for the finiteness of the film system. A second important observation is the 


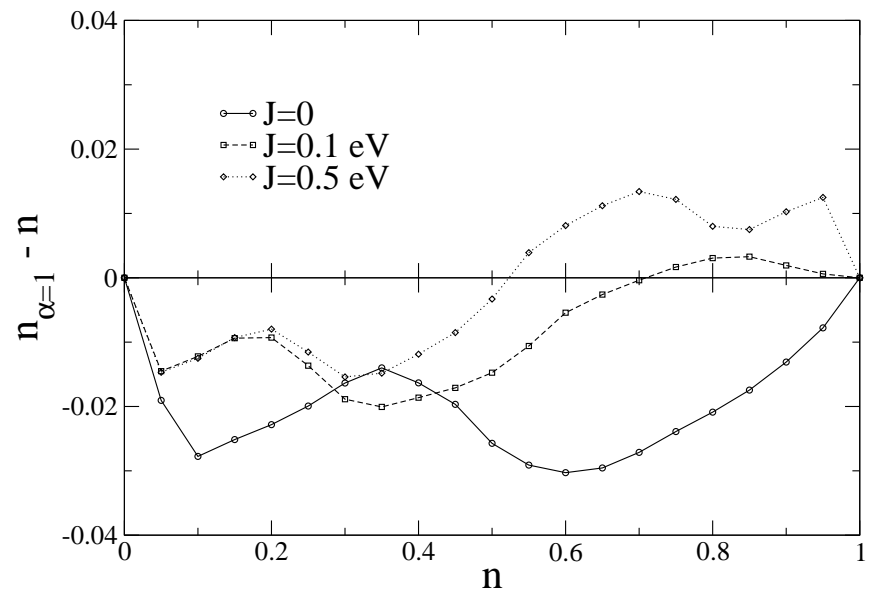

FIG. 2: Charge transfer in a 5-layer film at $T=0,\left\langle S_{z}\right\rangle=S$ (ferromagnetic saturation). Whereas the carrier density in the surface layer is always reduced in the non-interacting system there is an occupation above average for large enough $n$ in the correlated system.

reduced effective bandwidth of the surface compared to the center layer LDOS. This can be directly traced back to the lower variance of the surface LDOS: in the noninteracting limit $\Delta^{2} \rho_{0}=q t$ and $q_{\text {surface }} / q_{\text {bulk }}=5 / 6$ for a sc(100) geometry. The effect is thus not correlation induced and more pronounced for "open" film geometries. The band edges of the surface and center LDOS, however, are the same.

\section{Charge transfer}

The broken translational symmetry is furthermore responsible for the occurence of charge transfer, i.e. the deviation $\delta^{\alpha} n$ of the layer dependent band filling from the average occupation number:

$$
\delta^{\alpha} n=n_{\alpha}-n=\sum_{\sigma}\left(n_{\alpha \sigma}-\frac{1}{N_{L}} \sum_{\gamma} n_{\gamma \sigma}\right) .
$$

$N_{L}$ is the number of layers. Charge transfer is already present in the free electron film system and is due to the smaller effective bandwidth of the surface layer (occupation $n_{s}$ ). From this follows directly that in order to ensure thermodynamic equilibrium, i.e. a common chemical potential $\mu$, one has $n_{s}<n$ below and $n_{s}>n$ above half-filling $(n=1)$.

Switching on the coupling of the electron spins to the localized spins the question arises how this behavior changes. Fig. 2 shows the difference of the surface and the average occupation number as a function of $n$ at $T=0$ and ferromagnetic saturation. Whereas the electron density in the surface layer $n_{\alpha=1}$ is always below the average density $n$ in the non-interacting limit $(J=0)$ one also finds $\delta^{\alpha} n>0$ at $n<1$ for $J>0$. The explanation follows the same reasoning as above for the free system:

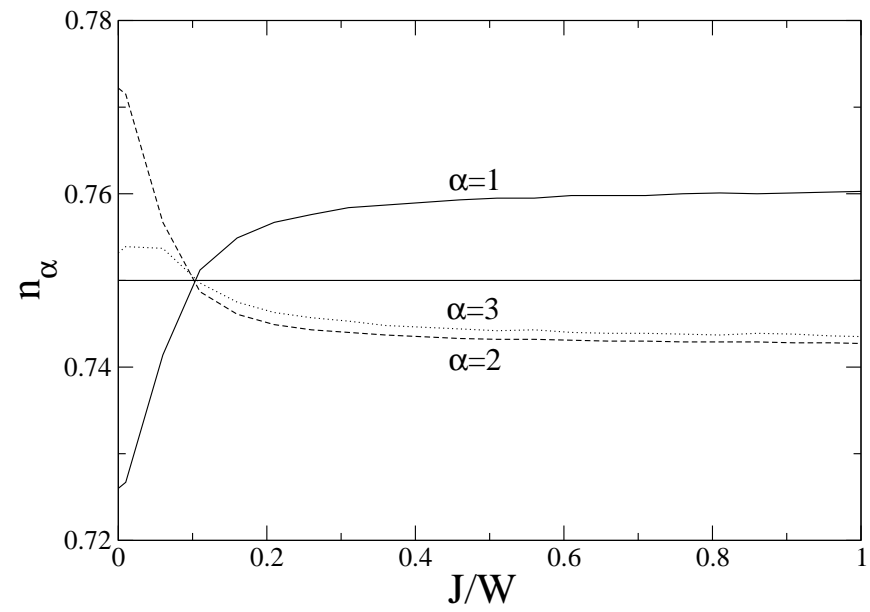

FIG. 3: Charge transfer in a 5-layer film at $T=0,\left\langle S_{z}\right\rangle=0$. The horizontal line at $n=0.75$ indicates the total average occupation number. The choice of the energy scale refers to the free simple cubic bulk bandwidth $W=12 t$.

a reduced or enhanced occupation in the surface layer is linked to the chemical potential being in the lower or upper half of the LDOS, respectively. As there is a (mainly $\downarrow-)$ transfer of spectral weight to higher energies for finite $J$ the chemical potential crosses the maximum of the lower band at values $n<1$. One finds the qualitatively same and quantitatively similar behavior in the spin disordered phase where $\left\langle S_{z}^{\alpha}\right\rangle=0$.

A second point is the dependence of the charge transfer on the coupling strength $J$. The interaction term (4) alone energetically favors single occupation of a lattice site for $n<1$. This can be easily seen in the zero bandwidth limit 25,35 It can thus be expected that a finite $J$ suppresses charge transfer. This is indeed the case as Fig. 3 shows where the electron density for the different layers of a 5-layer film is plotted as a function of the exchange coupling. The change of sign of $\delta^{\alpha} n$ is again caused by the same mechanism as pointed out above. Given a band occupation of $n<1$ the chemical potential moves from the lower half of the Bloch bands $(J=0)$ to the upper half of the lower quasiparticle subband $(J>0)$.

We observe that even $J \rightarrow \infty$ does not lead to complete charge neutrality and that at large $J$ the higher occupation of the surface layer goes with an almost homogeneous charge distribution in the inner layers of the film. It is also interesting to note that at $J \approx 0.1$ there is a quasi-homogenous charge distribution which coincides with the change of sign of $\delta^{\alpha} n$.

One can summarize that the effect of charge transfer is highest at low couplings $J$ and low average band occupations $n$, where it can amount to almost $30 \%$ (Fig. 2 at $n=0.1$ ). It appears to be of minor importance in the strong coupling regime, especially at higher charge carrier densities.

All the findings we have discussed in this section are qualitatively the same for other film thicknesses. As al- 


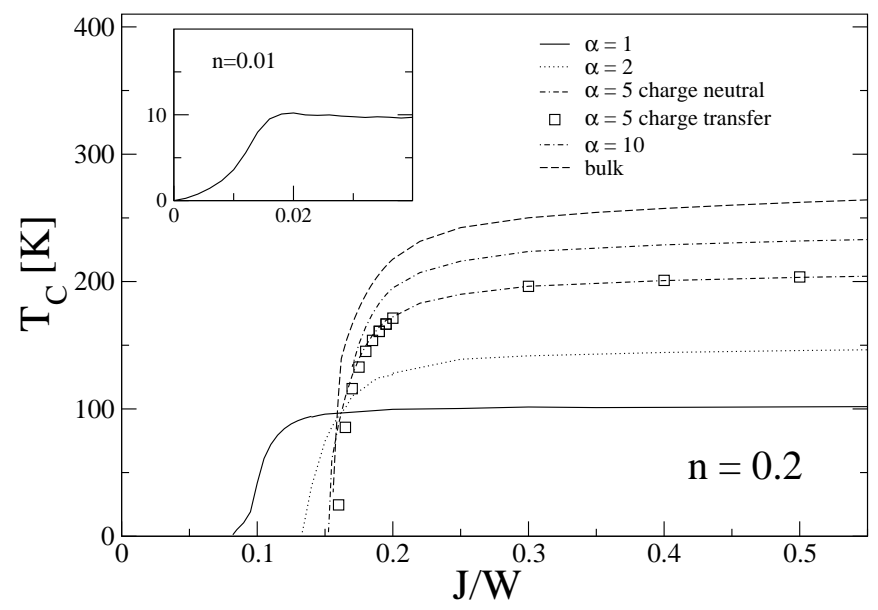

FIG. 4: Curie temperature for various numbers of layers. Parameters: $n=0.2, K_{2}=10^{-6} \mathrm{eV}$. There is no significant influence of charge transfer on the critical temperature. Inset: Curie temperature of a monolayer for a low band occupation.

ready mentioned charge transfer effects are more pronounced in "open" surface geometries with a stronger relative reduction in the number of nearest neighbors of a surface atom. However, the order of magnitude of the effect is the same.

Of course in real systems, Coulomb interaction prevents charge separation on a macroscopic scale. For the present purpose it is sufficient to discuss how charge transfer affects the ferromagnetic transition temperature of Kondo lattice films. To this end we will compare results based on calculations with and without charge transfer. Enforcing charge neutrality means we evaluated the layer-dependent centers of gravity of the Bloch bands self-consistently such that $n_{\alpha}=n$ for all $\alpha$. Where not otherwise stated the following results were obtained for charge neutral KLM films.

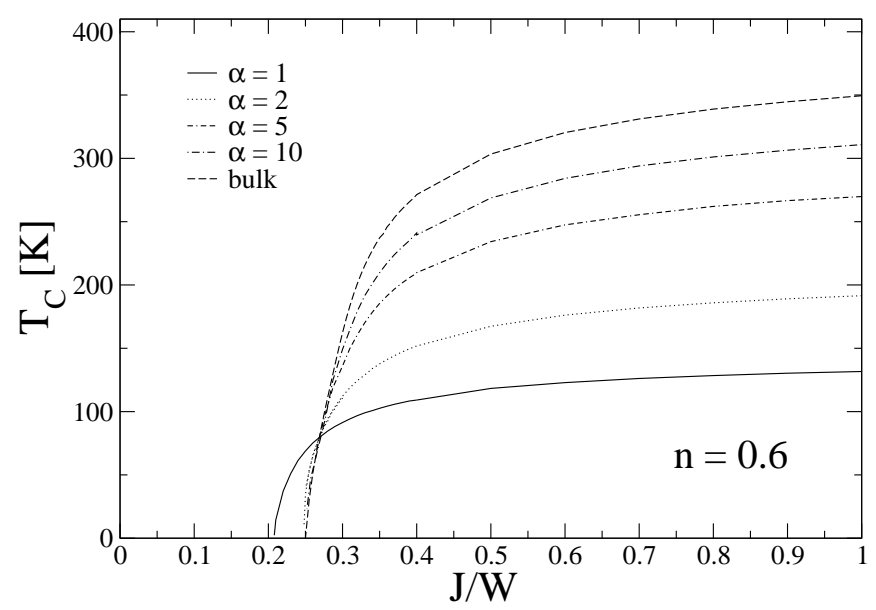

FIG. 5: Curie temperature for various numbers of layers. Parameters: $n=0.6, K_{2}=10^{-6} \mathrm{eV}$.

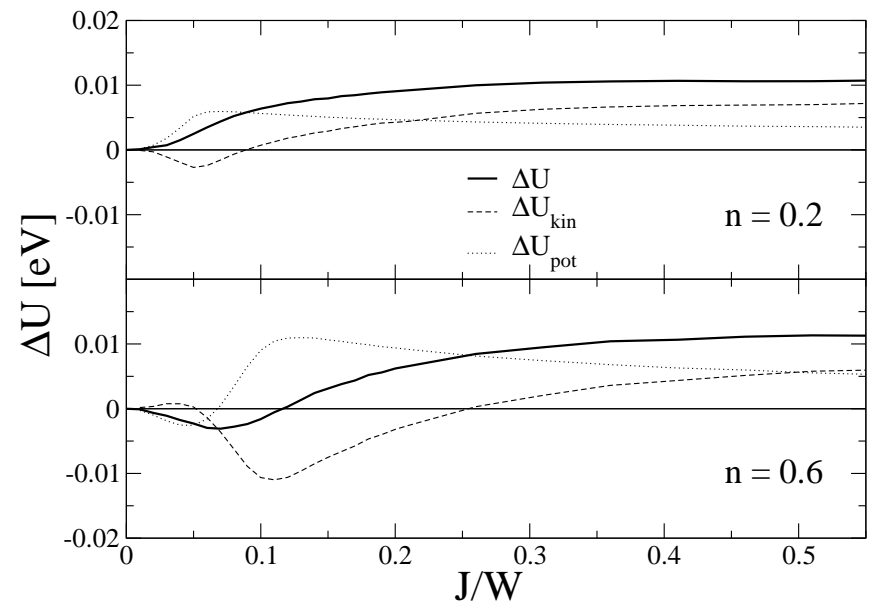

FIG. 6: Total, kinetic, and potential energy differences $\Delta U=$ $U_{\mathrm{PM}}(T=0)-U_{\mathrm{FM}}(T=0)$ between the paramagnetic and the ferromagnetic state as a function of the exchange coupling $J$ for a monolayer. The negative values of $\Delta U$ at small $J$ for $n=0.6$ indicate that ferromagnetism is unstable.

\section{B. Curie temperature}

\section{Whole J-range}

Our non-perturbative theory allows for an evaluation of KLM films at all coupling strengths $J$. In what follows the anisotropy constant is much smaller than the other energy scales in our model, $K_{2}<<W, J$. The influence of the anisotropy on the Curie temperature will be discussed in the last subsection in more detail.

Figs. 4 and 5 display the ferromagnetic critical temperature as a function of the exchange coupling $J$ for various film thicknesses. The inset in Fig. 4 shows $T_{C}$ of a monolayer for a small band occupation $n=0.01$. Here one recognizes the typical $T_{C} \sim J^{2}$-behavior of the perturbational RKKY interaction for small $J$. Increasing the electron density induces a critical interaction $J_{c}$ below which ferromagnetism is not stable. For a given number of layers $J_{c}$ increases with $n$. It has been shown before analytically that there is a minimum critical interaction strength for stable FM in the KLM in infinite dimensions $\frac{37}{37}$

To discuss this point further we consider the internal energy per site $U$ :

$$
U=\langle H\rangle=\left\langle H_{0}\right\rangle+\left\langle H_{J}\right\rangle=U_{\text {kin }}+U_{\text {pot }} .
$$

It can easily be calculated with the LDOS (6):

$$
U=\frac{1}{N_{L}} \sum_{\gamma \sigma} \int_{-\infty}^{+\infty} d E f(E) E \rho_{\gamma \sigma}(E) .
$$

We start from $F=U-T S$ where $F$ is the free energy and $S$ is the entropy. By comparing the difference of the internal energy at $T=0$ between the ferromagnetic $(\mathrm{FM})$ and paramagnetic $(\mathrm{PM})$ state, $\Delta U=$ 


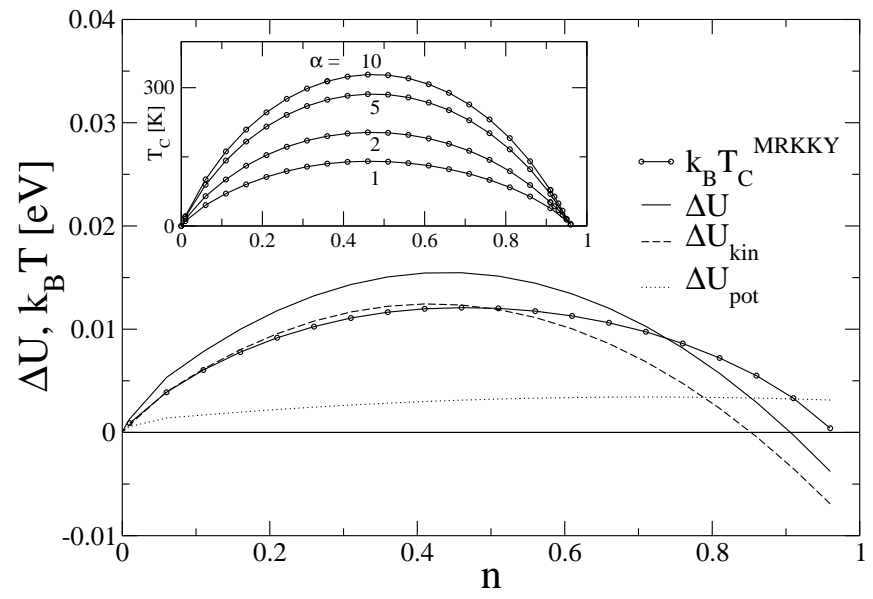

FIG. 7: Total, kinetic, and potential energy differences between the paramagnetic and the ferromagnetic state $\Delta U=$ $U_{\mathrm{PM}}\left(T=T_{C}\right)-U_{\mathrm{FM}}(T=0)$ as a function of the band occupation for a monolayer in the strong coupling regime. The Curie temperature obtained with the MRKKY theory is also included for comparison. Inset: MRKKY Curie temperature for various numbers of layers. Parameters: $J=1 \mathrm{eV}, K_{2}=$ $10^{-6} \mathrm{eV}$.

$U_{\mathrm{PM}}(T=0)-U_{\mathrm{FM}}(T=0)$, we can evaluate the stability of the ferromagnetic state against paramagnetism. In Fig. 6] the kinetic, potential, and total energy differences are shown for a monolayer at $T=0$. The magnetic stability for low to moderate $J$ is governed by a complex interplay between $U_{\text {kin }}$ and $U_{\text {pot }}$. Whereas at $n=0.2$ it is the potential energy which favors the (ferro)magnetic state it is both energies which make the ferromagnetic state unstable for higher $n$, leading to a critical interaction strength as in the self-consistent calculation based on the effective Heisenberg model before. The fact that in terms of the ground state energy there is no $J_{c}$ at $n=0.2$ contrary to the results in Fig. 4 suggests that other magnetic correlations such as non-commensurate or antiferromagnetic become important, reducing further the parameter range of FM stability.

Furthermore it is remarkable that the critical interaction strength is smallest for a monolayer whereas films with $\alpha>1$ behave rather bulk-like as far as $J_{c}$ is concerned. Taking into consideration the energy scales of the KLM one would expect this tendency. Quite generally the physics should be governed by the ratio $J / W$. Hence a smaller bandwidth due to a reduced number of layers implies a smaller $J$ around which the intermediate coupling regime is located. In addition the reduced bulk FM region might indicate favored antiferromagnetic configurations which are not existent in the 2D case for mere geometrical reasons.

For comparison we performed calculations where charge transfer was permitted. Allowing charge transfer means that we used equal center of gravities of the noninteracting local density of states $T_{0}^{\alpha}=T_{0}$ for all layers. We did not find any noteworthy influence of charge trans-

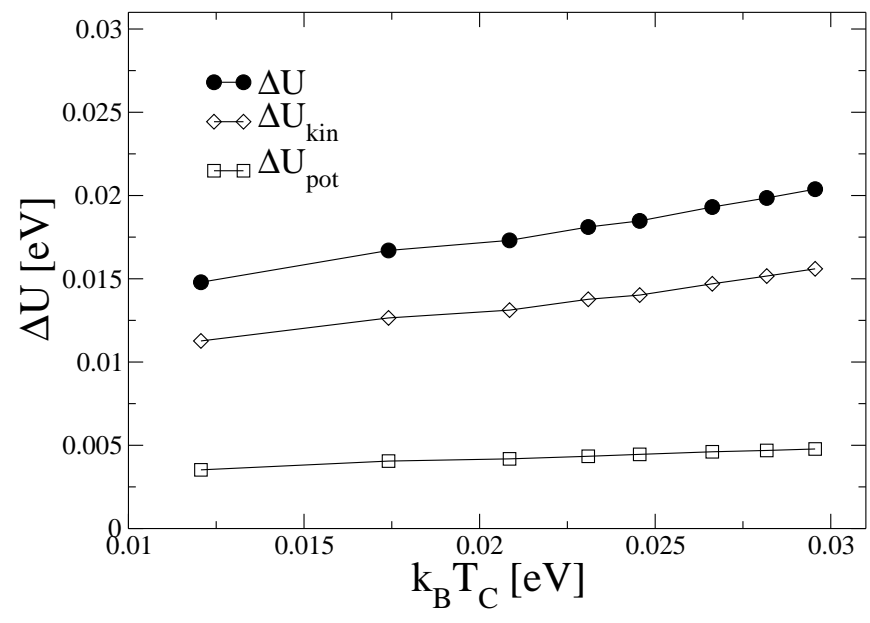

FIG. 8: Total, kinetic, and potential energy differences between the paramagnetic and the ferromagnetic state $\Delta U=$ $U_{\mathrm{PM}}\left(T=T_{C}\right)-U_{\mathrm{FM}}(T=0)$ as a function of the Curie temperature in the strong coupling regime. The film thickness is an implicit parameter and increases from left (monolayer) to right $\left(N_{L}=15\right)$. Parameters: $n=0.5, J=1 \mathrm{eV}, K_{2}=$ $10^{-6} \mathrm{eV}$.

fer on $T_{C}$ compared to the charge neutral case as Fig. 4 demonstrates for a 5-layer film $\frac{38}{2}$

\section{Double exchange regime}

In the strong coupling (double exchange) regime $(J S>>W)$ one observes that $T_{C}$ runs into saturation for large enough $J$ at all electron densities and numbers of layers. Once more we will contrast the results obtained by the MRRKY method with energy considerations. Whereas before we were interested in the stability of ferromagnetism at $T=0$ we want to be more quantitative in the following comparison of the Curie temperatures. Hence we evaluate the internal energy of the paramagnetic state at $T_{C}$ as the energy scale for the critical temperature is set by the change in total energy when going from the ferromagnetic to the paramagnetic state $T_{C} \sim \Delta U=\Delta U\left(T_{C}\right)=U_{\mathrm{PM}}\left(T=T_{C}\right)-U_{\mathrm{FM}}(T=0)$. In the double exchange regime $T_{C}$ is essentially determined by the kinetic energy: in the local frame the electron spin is oriented parallel to the localized spin and the potential energy does not change much between the ferromagnetic and the paramagnetic state; for $J \rightarrow \infty$ it stays the same. On the other hand hopping of electrons strongly depends on the magnetic configuration and is somehow blocked when the localized spins are disordered. For the bulk KLM the relationship between $T_{C}$ and $\Delta U_{\text {kin }}$ has already been analysed $\stackrel{37.39}{ }$

The inset of Fig. 7 shows the Curie temperature as a function of the band occupation $n$ for strong coupling. We obtain ferromagnetism for a wide range of $n$ up close to half-filling and a symmetry approximately around quarter-filling for all films. $T_{C}$ increases with the 


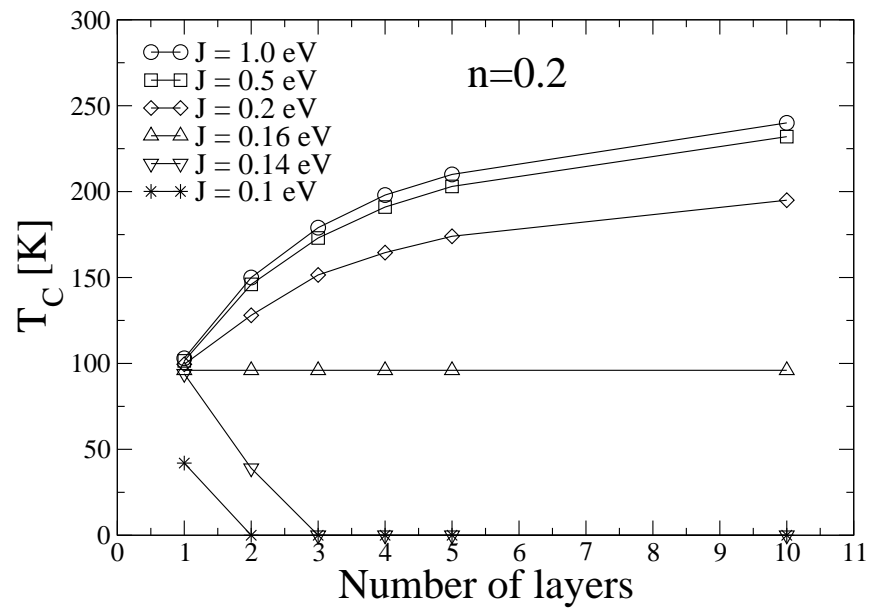

FIG. 9: Curie temperature as a function of the film thickness. Lines are guides to the eye. Parameters: $n=0.2, K_{2}=$ $10^{-6} \mathrm{eV}$.

number of layers. The maximum band occupation for which ferromagnetism exists does not depend on the film thickness, indicating that the FM phase boundary in the strong coupling regime is the same in $2 \mathrm{D}$ and $3 \mathrm{D}$. Also in Fig. [7 a comparison between $\Delta U$ and $T_{C}$ is shown. The variation of the critical temperature with $n$ is paralleled by a corresponding variation of $\Delta U_{\text {kin }} . \Delta U_{\text {pot }}$ varies only slowly with $n$. We find that, apart from a small difference in the maximum $n$ yielding ferromagnetism, there is even quantitatively a good agreement between the values of $T_{C}$ and $\Delta U$.

In Fig. 8 we show $\Delta U_{\text {kin }}$ and $\Delta U_{\text {pot }}$ as functions of $T_{C}$ with the number of layers as implicit parameter. One recognizes a linear dependence between the ferromagnetic transition temperature and the energy change. $T_{C}$ is again dominantly determined by the difference in kinetic energy of the FM and of the PM phase. We point out that the almost quantitative agreement of $T_{C}^{\mathrm{MRKKY}}$ and $\Delta U$ for the monolayer is due to the particular choice of the anisotropy parameter and thus rather coincidental. With an increasing number of layers deviations between the two quantities appear. However the magnitudes remain similar.

\section{3. $T_{C}$ as a function of film thickness}

We conclude this section by presenting the critical temperature as a function of the film thickness. As a natural consequence of the results and the discussion just presented this dependence varies drastically in the different coupling regimes as demonstrated in Fig. 9. Whereas for large $J$ the Curie temperature decreases when reducing the number of layers, reflecting the findings just discussed before in Fig. 8, we get a completely different picture for weak to intermediate strength of $J$. At $J=0.16$ the ferromagnetic transition temperature is equal for all films.

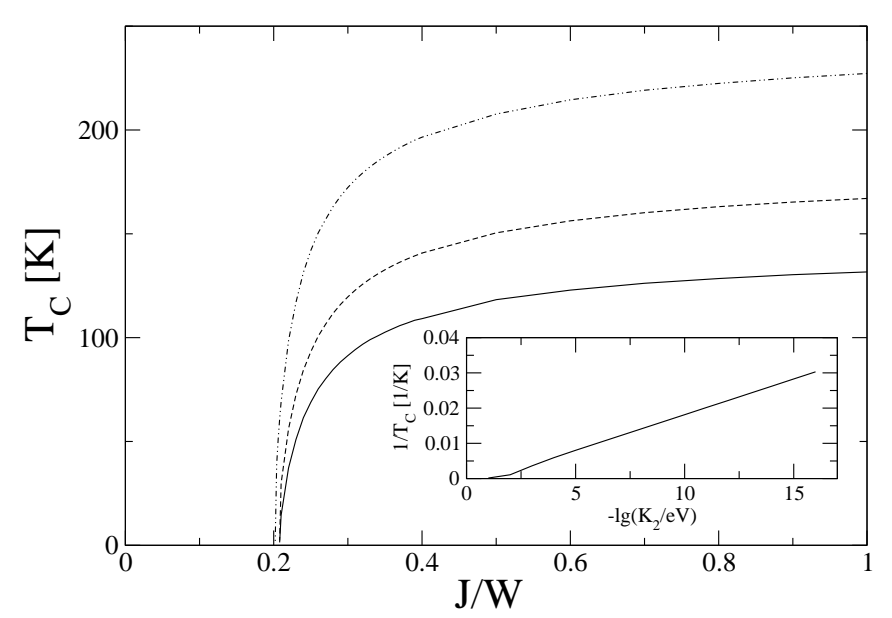

FIG. 10: Curie temperature of a monolayer at different anisotropy strengths for $n=0.6$ (from bottom to top: $K_{2}=$ $1,10,100 \mu \mathrm{eV})$. Varying the anisotropy has a considerable effect on $T_{C}$ in the strong coupling regime but only slightly changes $J_{c}$. Inset: Curie temperature as a function of $K_{2}$ for a monolayer in the saturation region $(n=0.2, J=0.2 \mathrm{eV}$, see also Fig. 4).

Reducing further the exchange coupling $J$ yields a finite $T_{C}$ only for very thin films.

\section{Anisotropy}

In the last subsection we want to briefly discuss the effect of a single-ion anisotropy on the ferromagnetic transition temperature of Kondo lattice films. There exists abundant literature on scaling relations between the critical temperature and key film parameters as anisotropy strength, exchange coupling, and the film thickness, mostly for Ising-like or Heisenberg films. As we are dealing with an effective localized spin model, derived from the KLM, these results should also apply in our case. The exchange integrals (14) now are, however, $T$-dependent. In the following investigation of the functional dependence $T_{C}\left(J, K_{2}\right)$ we restrict ourselves to a monolayer because in this case the role of anisotropy is most pronounced and the extension to multilayers is straightforward.

The effect of the anisotropy strength on the ferromagnetic transition temperature over the whole range of the intra-atomic exchange coupling $J$ is shown in Fig. 10, One can distinguish two regimes: As can be seen from the inset (representing the saturation regime) the well known logarithmic dependence of the Curie temperature on the anisotropy strength $K_{2}$ is also valid for the Kondo lattice model. Already a very small anisotropy is sufficient to obtain a $T_{C}$ which is of the order of magnitude of Curie temperatures realized for much larger, more realistic values of $K_{2}$.

Evaluating the formula for the Curie temperature (22) by solving the corresponding $q$-integral for the predomi- 
nantly contributing small wave vectors one gets

$$
\frac{1}{T_{C}} \propto \ln \left(1+c \frac{\tilde{J}_{T=T_{C}}}{K_{2}}\right) .
$$

$c$ is a constant $\approx 1$ and $\tilde{J}$ is the $T$-dependent effective coupling of one localized spin to all others, i.e. it determines the order of magnitude of the magnon energies, which are typically much larger than the anisotropy energy, $\tilde{J}>>K_{2}$. An analogous formula for the Heisenberg model with constant exchange coupling is discussed, e.g., in Ref. 40. In our case the $T$-dependence of the exchange integrals (14) in the paramagnetic regime is evidently too weak to cause significant deviations from the logarithmic dependence, not too surprisingly because this temperature dependence is a mere Fermi softening effect.

Whereas in the saturation region the scaling of $T_{C}$ with $K_{2}$ is determined by Eq. (27) as just discussed, in the critical region FM becomes unstable and a higher $K_{2}$ only slightly reduces $J_{c}$. There $T_{C} \rightarrow 0$ and thus the effective coupling $\tilde{J} \rightarrow 0$, see Eq. (22) where the effective coupling corresponds to the $\hat{J}$-exchange term. The critical region has a width of $\Delta J \approx 10^{-1} \mathrm{eV}$ within which the effective coupling typically changes by $\Delta \tilde{J} \approx 10^{-3} \mathrm{eV}$. Thus a $\Delta K_{2} \approx 10^{-4} \mathrm{eV}$ should shift $J_{c}$ by $\approx 10^{-2} \mathrm{eV}$, which is in accordance with the numerical findings in Fig. 10.

\section{CONCLUSIONS}

We have investigated ferromagnetic Kondo lattice films with a finite band occupation. Our self-consistent theory is made up by an electronic and a magnetic part. The one particle Green function is obtained within an equation of motion approach. Local moment correlation functions are calculated by an effective Heisenberg Hamiltonian which results from integrating out the electronic degrees of freedom.

We presented results for the temperature dependent one particle exciation spectrum and discussed charge transfer between film layers. Charge transfer occurs in all coupling regimes and, at strong coupling, leads to a pronounced deviation of the surface layer electron density from the rather homogeneous band occupation of the inner layers. The reduced dimensionality is also responsible for a reduced magnetization at the surface. However, $T_{C}$ is the same for all layers.
Curie temperatures were discussed for a variety of parameters like the exchange coupling $J$, the band occupation $n$, and for various number of layers. For large enough band occupation we obtain a critical $J_{c}$ below which ferromagnetism is not stable. In the strong coupling regime $T_{C}$ saturates and increases with the number of layers. We have not found any significant influence of charge transfer on the Curie temperature of KLM films.

In addition to the MRKKY self-consistent $T_{C}$-results energy calculations of the paramagnetic and ferromagnetic state were used to discuss both the critical interaction strength in the intermediate coupling regime and the crucial role the kinetic energy plays in the strong coupling regime. Depending on the values of the exchange coupling and the band occupation the dependence of the Curie temperature on the film thickness exhibits quite a different behavior. We also discussed the effects of a single-ion anisotropy on the ferromagnetic transition temperature. Whereas it can significantly elevate $T_{C}$ in the strong coupling regime it hardly affects the critical interaction strength $J_{c}$.

In this work we took the rather classical spin quantum number $S=7 / 2$. This would apply for rare earth systems as, e.g., Gd. Systems of current interest as DMS $(S=5 / 2)$ possess a somewhat high $S$, and even for the manganites with $S=3 / 2$ classical spins are believed to be a reasonable approximation at least at low temperatures. $\stackrel{41}{\underline{1}}$ Nevertheless a more profound investigation of the dependence of the magnetic properties on the spin quantum number at higher temperatures appears to be highly valuable.

A worthwhile extension of the present work would also be to use other lattice structures and to take additional magnetic configurations like antiferromagnetism into account. A combined bandstructure many-body calculation for Gd could contribute to the much debated issues of an enhanced surface Curie temperature and electronic surface states in this substance. More on the model level, making the exchange and anisotropy parameters layer dependent or analysing the influence of non-magnetic cap layers on the magnetic stability of KLM films would be interesting tasks. Furthermore an application to the problem of interlayer exchange coupling appears to be rewarding because the temperature dependent effective exchange integrals give direct access to the coupling between the layers in a film system. This investigation is planned for future work.
1 C. Zener, Phys. Rev. 81, 440 (1951); Phys. Rev. 82, 403 (1951)

2 P. W. Anderson and H. Hasegawa, Phys. Rev. 100, 675 (1955)

3 E. Dagotto, Nanoscale Phase Separation and Colossal Magnetoresistance, The Physics of Manganites and Related Compounds, Springer Series in Solid-State Sciences
136 (2003)

4 A. P. Ramirez, J. Phys. C 9, 8171 (1997)

${ }^{5}$ H. Ohno, J. Magn. Magn. Mat. 200, 110 (1999)

${ }^{6}$ I. Z̆utić, J. Fabian, and S. Das Sarma, Rev. Mod. Phys. 76, 323 (2004)

7 P. Wachter, Helv. Phys. Acta 37, 637 (1964)

${ }^{8}$ C. Santos, W. Nolting, and V. Eyert, Phys. Rev. B 69, 
$214412(2004)$

9 R. Schiller and W. Nolting, Phys. Rev. Lett. 86, 3847 (2001)

${ }^{10}$ H. Ott, S. J. Heise, R. Sutarto, Z. Hu, C. F. Chang, H. H. Hsieh, H.-J. Lin, C. T. Chen, and L. H. Tjeng, Phys. Rev. B 73, 094407 (2006)

11 S. Dhar, L. Perez, O. Brandt, A. Trampert, K. H. Ploog, J. Keller, and B. Beschoten, Phys. Rev. B 72, 245203 (2005)

12 A. A. Sidorenko, G. Allodi, R. De Renzi, G. Balestrino, and M. Angeloni, Phys. Rev. B 73, 054406 (2006)

13 K. Y. Wang, K. W. Edmonds, R. P. Campion, L. X. Zhao, C. T. Foxon, and B. L. Gallagher, Phys. Rev. B 72, 085201 (2005)

14 M. Farle and K. Baberschke in: Magnetism and Electronic Correlations in Local-moment Systems, M. Donath, P. A. Dowben, and W. Nolting (Eds.), p. 35, World Scientific (1998)

15 A. B. Shick, W. E. Pickett, and C. S. Fadley, Phys. Rev. B 61, R9213 (2000)

16 C. L. Nicklin, M. J. Everard, C. Norris, and S. L. Bennett, Phys. Rev. B 70, 235413 (2004)

17 J. Kienert and W. Nolting, J. Mag. Mag. Mat. 272-276, e887-e888 (2004)

18 A. Urbaniak-Kucharczyk, phys. stat. sol. (b) 186, 263 (1994)

19 W. Müller and W. Nolting, Phys. Rev. B 69, 155425 (2004)

${ }^{20}$ R. Schiller and W. Nolting, Phys. Rev. B 60, 462 (1999)

21 W. Nolting, S. Rex, and S. Mathi Jaya, J. Phys.: Condens. Matter 9, 1301 (1997)

22 C. Santos and W. Nolting, Phys. Rev. B 65, 144419 (2002)

${ }^{23}$ R. Schiller, Correlation Effects and Temperature Dependencies in Thin Ferromagnetic Films: Magnetism and Electronic Structure, Dissertation, Humboldt-Universität zu Berlin (2000)

${ }^{24}$ For a recent investigation of Kondo scaling in the ferromagnetic Kondo lattice see S. Biermann, L. de' Medici, and A. Georges, Phys. Rev. Lett. 95, 206401 (2005)

25 W. Nolting, Grundkurs Theoretische Physik 7, Viel-
Teilchen-Theorie, Springer Berlin (2005)

26 A. A. Rudermann and C. Kittel, Phys. Rev. 96, 99 (1954); T. Kasuya, Prog. Theor. Phys. 16, 45 (1956); K. Yosida, Phys. Rev. 106, 893 (1957)

27 W. Nolting, Quantentheorie des Magnetismus, Teubner Stuttgart (1986)

28 S. V. Tyablikov, Quantentheoretische Methoden des Magnetismus, Teubner Stuttgart (1969)

${ }^{29}$ H. B. Callen, Phys. Rev. 130, 890 (1963)

30 N. D. Mermin and H. Wagner, Phys. Rev. Lett. 17, 1133 (1966)

31 A. Gelfert and W. Nolting, J. Phys.: Condens. Matter 13, R505 (2001)

${ }^{32}$ F. B. Anderson and H. B. Callen, Phys. Rev. 136, A1068 (1964)

33 S. Schwieger, J. Kienert, and W. Nolting, Phys. Rev. B 71, 024428 (2005)

${ }^{34}$ For the Lines decoupling (M. E. Lines, Phys. Rev. 156, $534(1967)): \gamma=\frac{9}{2 S(S+1)}$

35 W. Nolting, J. Phys. C: Solid State Phys. 11, 1427 (1978)

36 The fact that a spin-spin interaction of RKKY-type is longranged does not affect the argument.

37 A. Chattopadhyay, A. J. Millis, and S. Das Sarma, Phys. Rev. B 61, 10738 (2000)

38 However we point out that the addition of Coulomb interactions, which are known to be relevant e.g. for manganites, can lead to a significant amount of charge transfer and a variety of magnetic phases that occur in superlattice structures, see a recent work of C. Lin, S. Okamoto, and A.J. Millis, Phys. Rev. B 73, 041104(R) (2006)

39 B. Michaelis and A. J. Millis, Phys. Rev. B 68, 115111 (2003)

40 P. Fröbrich, P.J. Jensen, and P.J. Kuntz, Eur. Phys. J. B 13, 477 (2000)

41 J. Kienert and W. Nolting, Phys. Rev. B 73, 224405 (2006) 